\title{
Effect of Continuous Nursing on Pain and Quality of Life in Patients with Rheumatoid Arthritis
}

\author{
Fang Fang, Li Liu, Pin Li, Guiyan Wen* \\ Department of Gastroenterology, The First Affiliated Hospital of Jinan University, Guangzhou 510000, China \\ *Corresponding author: Guiyan Wen, wengy78@126.com
}

\begin{abstract}
Objective: To explore the effectiveness of continuous nursing in the treatment of patients with rheumatoid arthritis for relieving pain and improving the quality of life of patients. Methods: A total of 80 patients with rheumatoid arthritis were treated in our department from January to August 2021. All patients were treated with continuous nursing, and their quality of life and pain related content were evaluated before admission examination, and their quality of life and pain after discharge were evaluated. Results: The pain degree of patients before continuous nursing intervention was $3.26 \pm 0.12$, and the pain degree score after nursing was $1.22 \pm 0.23, \mathrm{t}=70.3344, \mathrm{P}<0.05$. The difference is obvious and comparable; The scores of emotional function, role physical, social function, physical pain, physiological function, mental health, life vitality and overall health of patients before continuous nursing intervention were $71.33 \pm 1.23,72.12 \pm 1.36,71.56 \pm 1.42,73.25 \pm 2.01,74.36$ $\pm 0.96,73.12 \pm 2.45,72.98 \pm 1.63$ and $73.56 \pm 1.26$ respectively, and the scores of emotional function, role physical, social function, body pain, physiological function, mental health, life vitality and overall health were $91.66 \pm 1.03,93.23 \pm 1.36$, $92.69 \pm 1.06,93.69 \pm 1.47,92.98 \pm 1.69,95.36 \pm 1.23,94.01 \pm 1.66$ and $94.06 \pm 1.69$ respectively, $\mathrm{P}<0.05$. The differences are comparable. Conclusion: Through continuous nursing intervention, after rheumatoid patients are discharged from hospital, the degree of pain of patients is significantly reduced, and the quality of life is significantly improved. Patients can actively integrate into social life and maintain a good attitude.
\end{abstract}

Keywords: Continuous nursing; Rheumatoid arthritis; Quality of life

Publication date: November 2021; Online publication: November 30, 2021

\section{Introduction}

The incidence rate of rheumatoid arthritis in middle-aged group is high, and the performance is long, and the quality of life and pain are associated with the patients. The research shows that the incidence rate of this disease is $0.32 \%-0.36 \%$, while the rate of rheumatoid arthritis among middle-aged people aged 30-50 is $80 \%$. Rheumatoid arthritis is easy to relapse and affect the body of patients. And affect the mental health of patients ${ }^{[1-3]}$. Rheumatoid arthritis is a serious patient, which seriously affects the quality of life of patients. In this paper, continuous nursing was used to intervene patients with rheumatoid arthritis, and the quality of life and pain of patients after nursing intervention were analyzed.

\section{Materials and methods}

\subsection{General information}

Between January and August 2021, 80 patients with rheumatoid arthritis were treated in our department, including 43 male patients and 37 female patients. The age range was 31-62 years old, with an average age of $43.66 \pm 2.33$. Patients admitted to hospital were divided into two groups by random number method, with 40 patients in the control group received conventional care, and 40 patients in the study group received continuous care, and quality of life and pain related assessments were performed before admission. Before 
admission, the pain score of patients was $3.26 \pm 0.12$, and the scores of emotional functions, role physical, social function, physical pain, physiological function, mental health, life vitality and overall health were $71.33 \pm 1.23,72.12 \pm 1.36,71.56 \pm 1.42,73.25 \pm 2.01,74.36 \pm 0.96,73.12 \pm 2.45,72.98 \pm 1.63$ and 73.56 \pm 1.26 respectively.

\subsection{Methods}

The control group used routine nursing, and the study group used continuous nursing. The patients were discharged from hospital after being admitted to hospital three months ago, during which, the continuous nursing intervention was adopted for the patients, which can be seen as follows:

(1) Medication guidance. Provide medication guidance for patients, inform patients of the use, usage and dosage of each drug, so as to avoid wrong use by patients ${ }^{[4]}$.

(2) Discharge follow-up. Follow-up calls should be made regularly between one to three months after discharge, to check about the patient's situation and medication, guide the patient's medication methods and ways according to the patient's situation, and give professional answers when the patient has questions ${ }^{[5]}$.

(3) Psychological care. Patients should be informed that they can do some rehabilitation sports after discharge, be guided to maintain the affected parts and be leaded to return to normal life. Psychological states should also be accessed to guide the patients face realistic condition with a normal heart.

(4) Health education. Patients should be informed that they can exercise every day and guide the patients to carry out functional exercise. Patients should improve their body immunity, ensure good work and rest habits, develop healthy living habits, quit smoking, drink, light diet, etc. ${ }^{[2-5]}$.

\subsection{Observation indexes}

The degree of pain was assessed by NRs score; The quality of life score includes emotional function, role physical, social function, physical pain, physiological function, mental health, life vitality, overall health, etc. The full score is 100 , expressed by mean \pm standard deviation $(\bar{x} \pm s)$.

\subsection{Statistical methods}

The data of this study were processed by SPSS 20.0 statistical software. The measurement data is expressed by mean \pm standard deviation $(\bar{x} \pm s)$, and t-test is performed. The counting data is expressed by rate $(\%)$, and the line is $\chi^{2}$ inspection; If the test results show that $\mathrm{P}<0.05$, the difference is statistically significant.

\section{Results}

\subsection{Comparison of pain degree of patients before and after nursing}

As shown in Table 1., the pain degree of patients before continuous nursing intervention was $3.26 \pm 0.12$, and the pain degree score after nursing was $1.22 \pm 0.23, \mathrm{t}=70.3344, \mathrm{P}<0.05$. The difference is obvious and comparable.

\subsection{Comparison of patients' quality of life before and after nursing}

As shown in Table 2., the scores of emotional function, role physical, social function, physical pain, physiological function, mental health, life vitality and overall health of patients before continuous nursing intervention were $71.33 \pm 1.23,72.12 \pm 1.36,71.56 \pm 1.42,73.25 \pm 2.01,74.36 \pm 0.96,73.12 \pm 2.45,72.98$ \pm 1.63 and $73.56 \pm 1.26$ respectively, and the scores of emotional function, role physical, social function, body pain, physiological function, mental health, life vitality and overall health were $91.66 \pm 1.03,93.23 \pm$ $1.36,92.69 \pm 1.06,93.69 \pm 1.47,92.98 \pm 1.69,95.36 \pm 1.23,94.01 \pm 1.66$ and $94.06 \pm 1.69$ respectively, $\mathrm{P}$ $<0.05$. The differences are comparable. 
Table 1. Comparison of pain degree of patients before and after nursing ( $\bar{x} \pm \mathrm{s}$, points)

\begin{tabular}{llcc}
\hline Group & & Number of cases & Degree of pain \\
\hline Before continuous nursing intervention & 40 & $2.98 \pm 0.23$ \\
\hline \multirow{2}{*}{$\begin{array}{l}\text { After continuous nursing } \\
\text { intervention }\end{array}$} & Before continuous nursing intervention & 40 & $3.26 \pm 0.12$ \\
\cline { 2 - 4 } & After continuous nursing intervention & 40 & $1.22 \pm 0.23$ \\
\hline $\mathrm{t}$ & & & 70.3344 \\
\hline $\mathrm{P}$ & & & 0.0000 \\
\hline
\end{tabular}

Table 2. Comparison of patients' quality of life before and after nursing ( $\bar{x} \pm \mathrm{s}$, score)

\begin{tabular}{|c|c|c|c|c|c|}
\hline \multirow[b]{2}{*}{ Group $(\mathbf{n}=80)$} & \multirow{2}{*}{$\begin{array}{c}\text { Control } \\
\text { group } \\
(\mathrm{n}=40)\end{array}$} & \multicolumn{2}{|c|}{ Study group $(n=40)$} & \multirow[b]{2}{*}{$\mathbf{t}$} & \multirow[b]{2}{*}{$\mathbf{P}$} \\
\hline & & $\begin{array}{l}\text { Before continuous } \\
\text { nursing intervention }\end{array}$ & $\begin{array}{c}\text { After continuous } \\
\text { nursing intervention }\end{array}$ & & \\
\hline Emotional function & $79.12 \pm 1.36$ & $71.33 \pm 1.23$ & $91.66 \pm 1.03$ & 80.1457 & 0.0000 \\
\hline Role physical & $81.63 \pm 1.03$ & $72.12 \pm 1.36$ & $93.23 \pm 1.36$ & 69.4168 & 0.0000 \\
\hline Social function & $82.21 \pm 1.69$ & $71.56 \pm 1.42$ & $92.69 \pm 1.06$ & 75.4162 & 0.0000 \\
\hline Physical pain & $80.96 \pm 0.69$ & $73.25 \pm 2.01$ & $93.69 \pm 1.47$ & 51.9135 & 0.0000 \\
\hline Physiological function & $78.98 \pm 1.36$ & $74.36 \pm 0.96$ & $92.98 \pm 1.69$ & 60.5893 & 0.0000 \\
\hline Mental health & $83.54 \pm 2.36$ & $73.12 \pm 2.45$ & $95.36 \pm 1.23$ & 51.3084 & 0.0000 \\
\hline Life vitality & $84.12 \pm 1.45$ & $72.98 \pm 1.63$ & $94.01 \pm 1.66$ & 57.1703 & 0.0000 \\
\hline Overall health & $83.25 \pm 1.68$ & $73.56 \pm 1.26$ & $94.06 \pm 1.69$ & 61.5052 & 0.0000 \\
\hline
\end{tabular}

\section{Conclusion}

To sum up, through continuous nursing intervention, after rheumatoid patients are discharged from hospital, the degree of pain of patients is significantly reduced, and the quality of life is significantly improved. Patients can actively integrate into social life and maintain a good attitude.

\section{Disclosure statement}

The authors declare no conflict of interest.

\section{References}

[1] Yang JQ, 2020, Effect of Continuous Nursing on Pain and Quality of Life in Patients with Rheumatoid Arthritis. Journal of Clinic Nursing's Practicality, 5(09): 107.

[2] Li LY, 2019, Effect of Personalized Nursing on Pain and Quality of Life in Patients with Rheumatoid Arthritis. Clinical Medical \& Engineering, 26(10): 1417-1418.

[3] A WT, 2019, Analysis on Effects of Pain Nursing on Pain Symptoms and Quality of Life in Patients with Rheumatoid Arthritis. Chinese Community Doctors, 35(02): $163+165$.

[4] Yang QJ, Dong XJ, Li M, et al., 2018, Effect of Traditional Chinese Medicine Pain Nursing on Quality of Life in Patients with Rheumatoid Arthritis. Today Nurse (Early Issue), 25(07): 120-122.

[5] He XF, 2017, Effects Analysis of Pain Nursing on Quality of Life in Patients with Rheumatoid Arthritis. Journal of Clinic Nursing's Practicality, 2(52): 35-36. 